\title{
Synthesis of multisubstituted pyrroles via a CuI-catalyzed three- component coupling and a 1,8-diazabicyclo[5.4.0]undec-7-ene (DBU) / silica-gel promoted cyclization
}

\author{
Wanli Chen, ${ }^{*},{ }^{a}$ Lina Jin, ${ }^{a}$ Yinghong Zhu, ${ }^{b}$ and Weimin Mo ${ }^{a}$ \\ ${ }^{a}$ Center of Analysis \& Measurement, Zhejiang University of Technology, Hangzhou, \\ Zhejiang 310014, China \\ ${ }^{b}$ College of Chemical Engineering and Materials, Zhejiang University of Technology, \\ Hangzhou, \\ Zhejiang,310014, China \\ E-mail: chenwl@zjut.edu.cn
}

\begin{abstract}
Multisubstituted pyrroles with a 2-sulfonamido-group were synthesized through a CuI-catalyzed three-component coupling and a 1,8-diazabicyclo[5.4.0] undec-7-ene (DBU) / silica-gel promoted cyclization under mild conditions in moderate yields.
\end{abstract}

Keywords: Multisubstituted pyrroles, three-component coupling, 1,8-diazabicyclo[5.4.0]undec7-ene (DBU), silica-gel, cyclization

\section{Introduction}

Pyrroles are an important class of heterocycles due to their applications as bioactive compounds and synthetic intermediates in organic synthesis. ${ }^{1}$ Recently it was also found that pyrroles have broad application in the field of materials chemistry and as structural elements in molecular recognition studies. ${ }^{2}$ Classical methods for the synthesis of these nitrogen heterocycles include the Knorr, ${ }^{3}$ Hantzsch, ${ }^{4}$ and Paal-Knorr ${ }^{5}$ reactions. ${ }^{6}$ However, these approaches usually present some limitations in terms of substituents that can be introduced, the substitution pattern, or regioselectivity. As a result, development of new and efficient methodologies for the synthesis of pyrroles with different substituent groups from simple, readily available starting materials remains an important research theme in organic chemistry though several novel synthetic strategies have been described in recent years. ${ }^{7}$

Recently, Fokin et al. have reported a CuI-catalyzed coupling reaction of terminal alkynes and sulfonyl azides to intermediate ketenimine derivatives, generated in situ from the triazole 
cycloadduct upon release of $\mathrm{N}_{2}$ (Scheme 1), ${ }^{8}$ which could be further transformed to some interesting heterocycles in a one-pot fashion. ${ }^{9}$

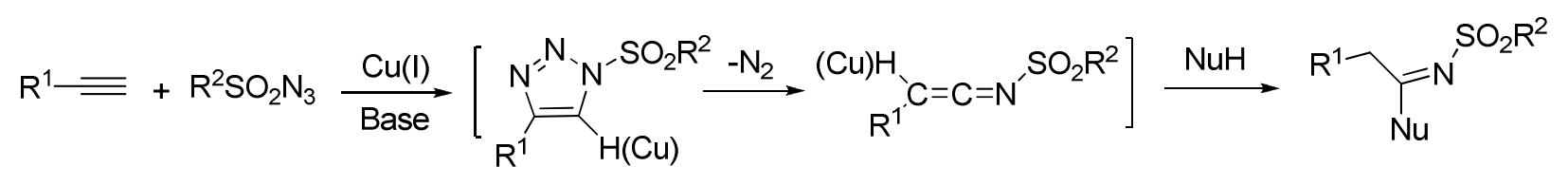

\section{Scheme 1}

In these reactions, the ketenimines trapped firstly by various nucleophiles is the key step. It was envisioned that this kind of ketenimines might be used as an efficient structure unit in the construction of pyrroles (Figure 1). Retrosynthetically, 2-tosylaminopyrrole derivatives could be prepared by scission of the bond between C-3 and C-4 of the pyrrole to the acetamidines containing a carbonyl group, which in turn could be obtained conveniently by a three-component reaction using sulfonyl azide, terminal alkyne and amino ketone. In our continual efforts to explore the synthesis of heterocyclic compounds, ${ }^{10}$ herein we present a CuI-catalyzed threecomponent coupling and a DBU / silica-gel promoted cyclization to afford an efficient synthesis of multisubstituted pyrroles with a 2-tosylamino-group function, ${ }^{11}$ which would be useful intermediates for access to other heterocyclic derivatives since they could be further elaborated to amplify complexity via a variety of carbon-carbon or carbon-heteroatom bond formation reactions. $^{12}$
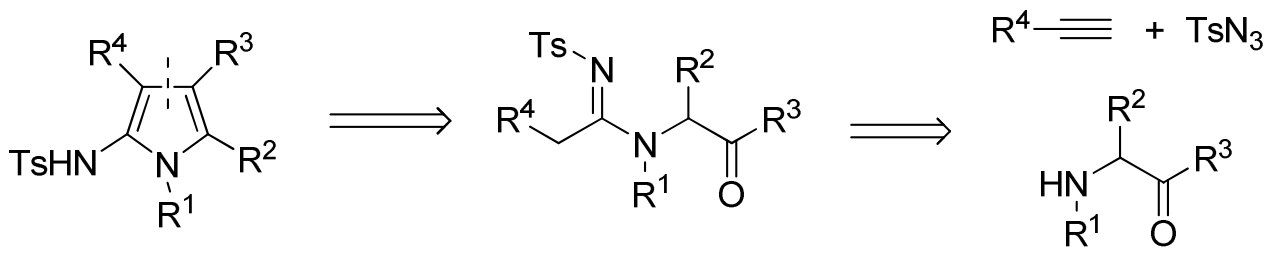

Figure 1. Synthetic strategy.

\section{Results and Discussion}

In our primary investigations, we selected the CuI-catalyzed reaction of $p$-toluenesulfonyl azide (1a), phenylacetylene (2a), 2-(phenylamino)-1-phenylethanone (3a) as the model reaction (Table 1). After workup and isolation, the desired product $\mathbf{4} \mathbf{a}^{13}$ was obtained in $17 \%$ yield. In order to optimize the reaction conditions, several solvent and bases were examined. THF was found to be the suitable solvent for this transformation in comparison with others, such as $\mathrm{CH}_{3} \mathrm{CN}$, DMF and $\mathrm{CHCl}_{3}$ (Table 1, entries 1-4). A lower yield was obtained when pyridine was used as the base (Table 1, entry 5). 
Table 1. Optimization of reaction conditions for the formation $\mathbf{4} \mathbf{a}^{\mathrm{a}}$

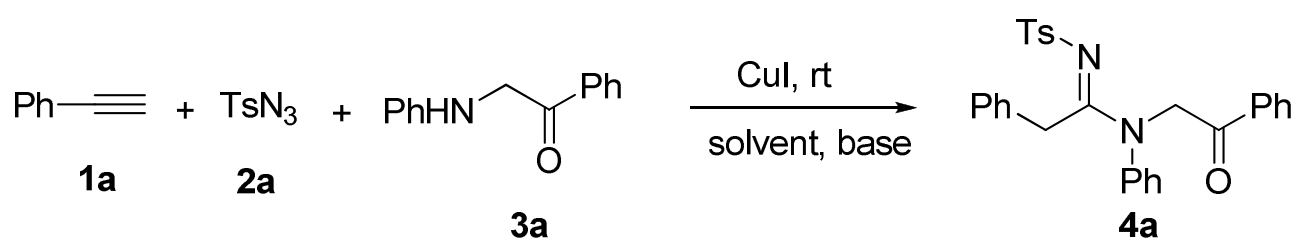

\begin{tabular}{ccccc}
\hline Entry & Solvent & Base & $\mathrm{t}(\mathrm{h})$ & ${\text { Yield }(\%)^{\mathrm{b}}}^{\text {be }}$ \\
\hline 1 & $\mathrm{CH}_{3} \mathrm{CN}$ & $\mathrm{Et}_{3} \mathrm{~N}$ & 5 & 17 \\
2 & $\mathrm{DMF}$ & $\mathrm{Et}_{3} \mathrm{~N}$ & 4 & 42 \\
3 & $\mathrm{CHCl}_{3}$ & $\mathrm{Et}_{3} \mathrm{~N}$ & 20 & 50 \\
4 & $\mathrm{THF}$ & $\mathrm{Et}_{3} \mathrm{~N}$ & 5 & 78 \\
5 & $\mathrm{THF}$ & $\mathrm{Py}$ & 24 & 66 \\
\hline
\end{tabular}

${ }^{\mathrm{a}}$ Reaction conditions: 1a $(1.3 \mathrm{mmol}), \mathbf{2 a}(1.3 \mathrm{mmol}), \mathbf{3 a}(1.0 \mathrm{mmol})$, solvent $(2 \mathrm{~mL}), \mathrm{Et}_{3} \mathrm{~N}(1.0$ mmol), CuI (0.1 mmol). ${ }^{\mathrm{b}}$ Isolated yield based on 3a.

We then investigated the possibility for the cyclization of $\mathbf{4 a}$ using 1,8diazabicyclo[5.4.0]undec-7-ene (DBU) as the base. In an initial experiment, we observed the formation of pyrrole 5a in 57\% yield when the reaction was performed in $\mathrm{CH}_{3} \mathrm{CN}$ using DBU as the base (Table 2, entry 1). Then we tried to screen the reaction conditions to improve the yield. To our delight, we found that silica-gel could promote this transformation. ${ }^{14}$ A more competitive yield was obtained when DBU(3 equiv.) was used (Table 2, entries 2-5). Next, we examined the effect of solvent choice on this reaction (Table 2, entries 6-9). A better yield was obtained when $\mathrm{CH}_{3} \mathrm{CN}$ was used as the solvent (Table 2, entry 4).

Table 2. Optimization of reaction conditions for the cyclization $\mathbf{4} \mathbf{a}^{\mathrm{a}}$
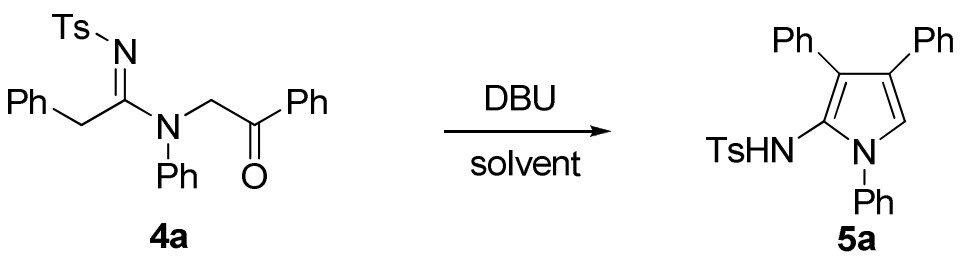

\begin{tabular}{cccc}
\hline Entry & Solvent & $\mathrm{t}(\mathrm{h})$ & ${\text { Yield }(\%)^{\mathrm{b}}}^{\mathrm{c}}$ \\
\hline $1^{\mathrm{c}}$ & $\mathrm{CH}_{3} \mathrm{CN}$ & 8 & 57 \\
2 & $\mathrm{CH}_{3} \mathrm{CN}$ & 12 & 0 \\
$3^{\mathrm{d}}$ & $\mathrm{CH}_{3} \mathrm{CN}$ & 5 & 72 \\
$4^{\mathrm{e}}$ & $\mathrm{CH}_{3} \mathrm{CN}$ & 5 & 79 \\
\hline
\end{tabular}


Table 2. Continued

\begin{tabular}{cccc}
\hline Entry & Solvent & $\mathrm{t}(\mathrm{h})$ & ${\text { Yield }(\%)^{\mathrm{b}}}^{\mathrm{b}}$ \\
\hline $5^{\mathrm{f}}$ & $\mathrm{CH}_{3} \mathrm{CN}$ & 5 & 76 \\
$6^{\mathrm{e}}$ & $\mathrm{C}_{2} \mathrm{H}_{5} \mathrm{OH}$ & 6 & 55 \\
$7^{\mathrm{e}}$ & DMSO & 8 & 43 \\
$9^{\mathrm{e}}$ & $\mathrm{THF}$ & 15 & 76 \\
\hline
\end{tabular}

${ }^{\mathrm{a}}$ Reaction conditions: 4a $(0.3 \mathrm{mmol})$, solvent $(2 \mathrm{~mL})$, DBU $(0.3 \mathrm{mmol})$, silica-gel $(0.5 \mathrm{~g}){ }^{\mathrm{b}}$ Isolated yield based on $\mathbf{4 a} .{ }^{\mathrm{c}}$ no silica-gel was added. ${ }^{\mathrm{d}} 2$ equiv. DBU was added. ${ }^{\mathrm{e}} 3$ equiv. DBU was added. ${ }^{\mathrm{f}} 4$ equiv. DBU was added.

With the optimized reaction conditions in hand (Table 2, entry 4), we further examined the scope of the reaction and the results were summarized in Table 3. From the results in Table 3, we could see that the reaction could proceed smoothly to afford pyrroles in moderate to good yields. Lower yields were obtained when $\mathrm{R}^{4}$ or $\mathrm{R}^{3}$ were alkyl group relative to aryl group (Table 3 , entries 5-6). Tetrasubstituted pyrrole could also be obtained in moderate yield (Table 3, entry $11)$.

Table 3. Cyclization of $\mathbf{4}$ to pyrroles $\mathbf{5}^{\mathrm{a}}$

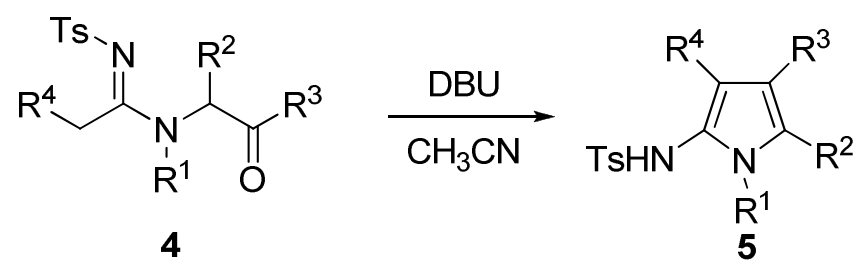

\begin{tabular}{ccccc}
\hline Entry & $\mathrm{R}^{1} / \mathrm{R}^{2} / \mathrm{R}^{3} / \mathrm{R}^{4}$ & Time $(\mathrm{h})$ & Product & \multicolumn{2}{c}{${\text { Yield }(\%)^{a}}^{a}$} \\
\hline 1 & $\mathrm{Ph} / \mathrm{H} / \mathrm{Ph} / \mathrm{Ph}, \mathbf{4 a}$ & 6 & $\mathbf{5 a}$ & 79 \\
2 & $p-\mathrm{CH}_{3} \mathrm{C}_{6} \mathrm{H}_{4} / \mathrm{H} / \mathrm{Ph} / \mathrm{Ph}, \mathbf{4 b}$ & 8 & $\mathbf{5 b}$ & 78 \\
3 & $o-\mathrm{CH}_{3} \mathrm{C}_{6} \mathrm{H}_{4} / \mathrm{H} / \mathrm{Ph} / \mathrm{Ph}, \mathbf{4 c}$ & 8 & $\mathbf{5 c}$ & 75 \\
4 & $\mathrm{Ph} / \mathrm{H} / p-\mathrm{CH}_{3} \mathrm{C}_{6} \mathrm{H}_{4} / \mathrm{Ph}, \mathbf{4 d}$ & 6 & $\mathbf{5 d}$ & 83 \\
5 & $\mathrm{Ph} / \mathrm{H} / \mathrm{CH}_{3} / \mathrm{Ph}, \mathbf{4 e}$ & 8 & $\mathbf{5 e}$ & 50 \\
6 & $\mathrm{Ph} / \mathrm{H} / \mathrm{Ph} / n-\mathrm{C}_{5} \mathrm{H}_{11}, \mathbf{4 f}$ & 22 & $\mathbf{5 f}$ & 27 \\
7 & $\mathrm{Ph} / \mathrm{H} / \mathrm{Ph} / p-\mathrm{CH}_{3} \mathrm{C}_{6} \mathrm{H}_{4}, \mathbf{4 g}$ & 8 & $\mathbf{5 g}$ & 61 \\
8 & $\mathrm{Ph} / \mathrm{H} / \mathrm{Ph} / m-\mathrm{ClC}_{6} \mathrm{H}_{4}, \mathbf{4 h}$ & 6 & $\mathbf{5 h}$ & 75 \\
9 & $\mathrm{Ph} / \mathrm{H} / p-\mathrm{CH} \mathrm{C}_{6} \mathrm{H}_{4} / m-\mathrm{ClC}_{6} \mathrm{H}_{4}, \mathbf{4 i}$ & 6 & $\mathbf{5 i}$ & 89 \\
10 & $\mathrm{Ph} / \mathrm{CH}_{3} / \mathrm{Ph} / m-\mathrm{ClC}_{6} \mathrm{H}_{4}, \mathbf{4 j}$ & 5 & $\mathbf{5 j}$ & 56 \\
\hline
\end{tabular}

${ }^{a}$ Isolated yield based on 4 . 
In order to simply the manipulation, then we tried to obtain 5a in a one-pot, sequential procedure. Using THF for the first step and $\mathrm{CH}_{3} \mathrm{CN}$ for the second step, we obtained 5a in $60 \%$ yield.

Plausible reaction mechanism. On the basis of the above results, a plausible mechanism for the DBU / silica-gel promoted cyclization was outlined in Scheme 2. Firstly, the deprotonation with DBU produces the carbanion A. Subsequent nucleophilic attack of the intramolecular carbonyl group and capture of a hydrogen cation to afford alcohol $\mathbf{B} .{ }^{14}$ Then elimination of one molecular water and aromatization of the resulting intermediate to afford pyrroles $\mathbf{5}$.
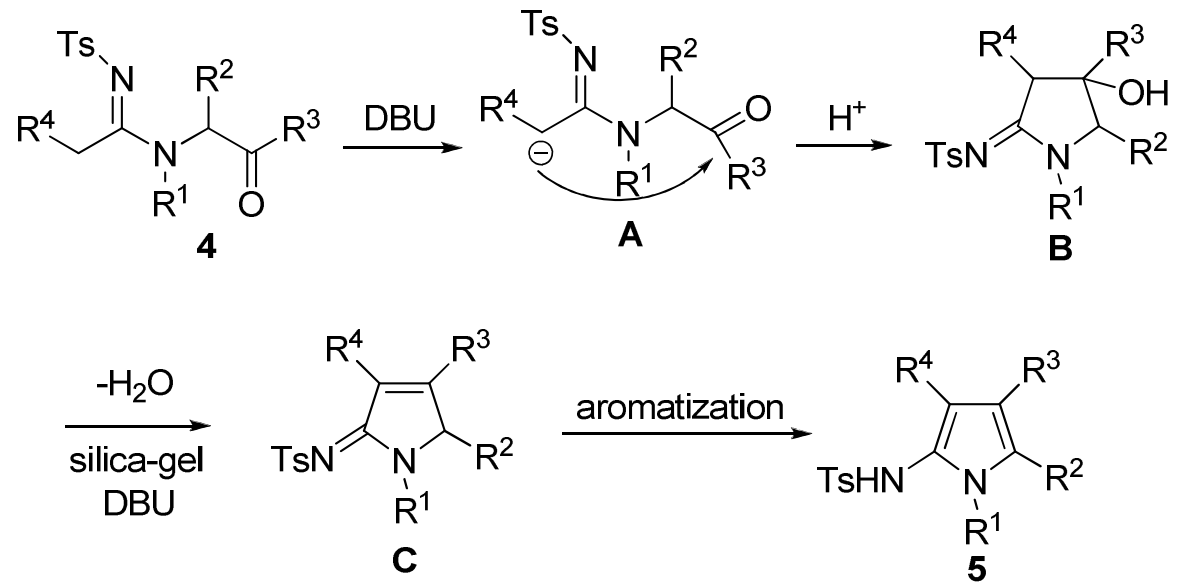

\section{Scheme 2}

\section{Conclusion}

In summary, we have developed an efficient method for the synthesis of multisubstituted pyrroles with a 2-tosylamino-group function in moderate to good yields under mild conditions. These pyrrole compounds bearing a 2-tosylamino functional group may be converted to other interesting and useful structural units in organic synthesis. Further studies into the scope and synthetic applications of this transformation are being carried out in our laboratory.

\section{Experimental Section}

General. All ${ }^{1} \mathrm{H}$ - and ${ }^{13} \mathrm{C}$ - NMR spectra were measured in $\mathrm{CDCl}_{3}\left(\mathrm{DMSO}-d_{6}\right)$ and recorded on Bruker Avance III $500 \mathrm{MHz}(125 \mathrm{MHz})$ spectrometer spectra with TMS as the internal standard. Chemical shifts are expressed in ppm and $J$ values are given in Hz. HRMS were performed on a Agilent 6210 LC/TOF instrument. IR spectra were run on a Thermo Nicolet 6700 spectrometer. 
Melting points were measured using CRC-1 melting point instrument and are uncorrected. Solvents were distilled before use. 2-(Arylamino)-1-arylethanones and 1-(phenylamino)- propan2-one were prepared as previously described. ${ }^{15}$

\section{General procedure for the synthesis of 4}

To a mixture of CuI $(0.1 \mathrm{mmol})$, sulfonyl azide $2(1.3 \mathrm{mmol})$, alkyne $1(1.3 \mathrm{mmol})$ and 2(arylamino)-1-arylethanones or 1-(phenylamino)propan-2-one $(1.0 \mathrm{mmol})$ in $\mathrm{THF}(2 \mathrm{~mL})$ was added $\mathrm{Et}_{3} \mathrm{~N}$ ( $1 \mathrm{mmol}$ ) under an $\mathrm{N}_{2}$ atmosphere at room temperature. The mixture was stirred until reaction was completed (monitored by TLC) and then evaporated on vacuum. The residue was subjected to silica gel column chromatography with petroleum ether/ethyl acetate as eluent.

(E)- $\boldsymbol{N}$-(2-Oxo-2-phenylethyl)- $\boldsymbol{N}$,2-diphenyl- $\boldsymbol{N}^{\prime}$-tosylacetamidine (4a). Solid (mp 122-124 ${ }^{\circ} \mathrm{C}$ ). ${ }^{1} \mathrm{H}$ NMR $\left(500 \mathrm{MHz}, \mathrm{CDCl}_{3}\right) \delta 7.78(\mathrm{~d}, 2 \mathrm{H}, J 7.7 \mathrm{~Hz}, \mathrm{Ar}), 7.54-7.51(\mathrm{~m}, 3 \mathrm{H}, \mathrm{Ar}), 7.38(\mathrm{t}, 2 \mathrm{H}, J$ 7.8 Hz, Ar), 7.25-7.19 (m, 3H, Ar), 7.12-7.09 (m, 5H, Ar), 6.96 (d, 2H, J 8.1 Hz, Ar), 6.94-6.92 (m, 2H, Ar), 5.05 (s, 2H, - $\left.\mathrm{CH}_{2}\right), 4.30\left(\mathrm{~s}, 2 \mathrm{H},-\mathrm{CH}_{2}\right), 2.29\left(\mathrm{~s}, 3 \mathrm{H},-\mathrm{CH}_{3}\right) .{ }^{13} \mathrm{C}-\mathrm{NMR}(125 \mathrm{MHz}$, $\left.\mathrm{CDCl}_{3}\right): \delta 192.7,166.3,142.0,141.8,140.3,135.1,134.4,133.5,129.5,128.8,128.6,128.2$, 128.1, 127.9, 126.4, 126.2, 59.0, 37.0, 21.4; IRv $\max \left(\mathrm{cm}^{-1}\right): 3032,1700,1530,1144,1091,763$, 693; HRMS-ESI: $[\mathrm{M}+\mathrm{H}]^{+} \mathrm{m} / z$ calcd for $\mathrm{C}_{29} \mathrm{H}_{27} \mathrm{~N}_{2} \mathrm{O}_{3} \mathrm{~S}$ : 483.1742 found: 483.1730 .

(E)- $\boldsymbol{N}$-(2-Oxo-2-phenylethyl)-2-phenyl- $\boldsymbol{N}$-p-tolyl- $\boldsymbol{N}$ '-tosylacetamidine (4b). Solid (mp 168$\left.170{ }^{\circ} \mathrm{C}\right) .{ }^{1} \mathrm{H}$ NMR $\left(500 \mathrm{MHz}, \mathrm{CDCl}_{3}\right) \delta$ 7.82-7.80 (m, 2H, Ar), 7.60-7.56 (m, 1H, Ar), $7.51(\mathrm{~d}, J$ $8.2 \mathrm{~Hz}, 2 \mathrm{H}, \mathrm{Ar}$ ), $\delta 7.42$ (t, J 8.0 Hz, 2H, Ar), 7.16-7.15 (m, 3H, Ar), 7.05-6.98 (m, 8H, Ar), 5.08 $\left(\mathrm{s}, 2 \mathrm{H},-\mathrm{CH}_{2}\right), 4.32\left(\mathrm{~s}, 2 \mathrm{H},-\mathrm{CH}_{2}\right), 2.32\left(\mathrm{~s}, 3 \mathrm{H},-\mathrm{CH}_{3}\right), 2.31\left(\mathrm{~s}, 3 \mathrm{H},-\mathrm{CH}_{3}\right) .{ }^{13} \mathrm{C}-\mathrm{NMR}(125 \mathrm{MHz}$, $\left.\mathrm{CDCl}_{3}\right): \delta 192.8,166.4,141.7,140.3,139.5,138.8,135.2,134.5,133.4,130.0,128.7,128.6$, 128.2, 127.9, 127.7, 126.3, 126.2, 59.1, 36.8, 21.4, 21.0; IRv $v_{\max }\left(\mathrm{cm}^{-1}\right): 3034,1699,1535,1145$, 1091, 743; HRMS-ESI: [M+H] $]^{+} \mathrm{m} / z$ calcd for $\mathrm{C}_{30} \mathrm{H}_{29} \mathrm{~N}_{2} \mathrm{O}_{3} \mathrm{~S}: 497.1899$ found: 497.1889 .

(E)- $\boldsymbol{N}$-(2-Oxo-2-phenylethyl)-2-phenyl- $\boldsymbol{N}$-o-tolyl- $\boldsymbol{N}^{\prime}$-tosylacetamidine (4c)

Solid (mp 124-126 ${ }^{\circ} \mathrm{C}$ ). ${ }^{1} \mathrm{H}$ NMR (500 MHz, $\left.\mathrm{CDCl}_{3}\right) \delta$ 7.81-7.79 (m, 2H, Ar), 7.59-7.54 (m, 3H, Ar), 7.44-7.40 (m, 3H, Ar), 7.28-7.23 (m, 1H, Ar), 7.16-7.10 (m, 5H, Ar), 7.00 (d, J 8.2 Hz, 2H, Ar), 6.91-6.89 (m, 2H, Ar), $5.79\left(\mathrm{~d}, J 16.6 \mathrm{~Hz}, 1 \mathrm{H},-\mathrm{CH}_{2}\right), 4.40\left(\mathrm{~d}, J 14.7 \mathrm{~Hz}, 1 \mathrm{H},-\mathrm{CH}_{2}\right), 4.16$ (d, $\left.J 14.7 \mathrm{~Hz}, 1 \mathrm{H},-\mathrm{CH}_{2}\right), 4.10\left(\mathrm{~d}, J 16.6 \mathrm{~Hz}, 1 \mathrm{H},-\mathrm{CH}_{2}\right), 2.33\left(\mathrm{~s}, 3 \mathrm{H},-\mathrm{CH}_{3}\right), 1.77\left(\mathrm{~s}, 3 \mathrm{H},-\mathrm{CH}_{3}\right) .{ }^{13} \mathrm{C}-$ NMR $\left(125 \mathrm{MHz}, \mathrm{CDCl}_{3}\right)$ : $\delta 192.4,166.4,141.7,140.7,140.4,136.0,135.2,133.7,133.4,131.2$, 129.5, 129.1, 128.8, 128.6, 128.2, 127.9, 127.0, 126.6, 126.2, 57.8, 36.8, 21.4, 17.0; IR $v_{\max }\left(\mathrm{cm}^{-}\right.$ $\left.{ }^{1}\right)$ : 3028, 1696, 1530, 1145, 1087, 743, 720; HRMS-ESI: $[\mathrm{M}+\mathrm{H}]^{+} \mathrm{m} / z$ calcd for $\mathrm{C}_{30} \mathrm{H}_{29} \mathrm{~N}_{2} \mathrm{O}_{3} \mathrm{~S}$ : 497.1899 found: 497.1920.

(E)- $\boldsymbol{N}$-(2-Oxo-2-p-tolylethyl)- $N$,2-diphenyl- $\boldsymbol{N}^{\prime}$-tosylacetamidine (4d). Solid (mp 156-158 $\left.{ }^{\circ} \mathrm{C}\right)$. ${ }^{1} \mathrm{H}$ NMR $\left(500 \mathrm{MHz}, \mathrm{CDCl}_{3}\right) \delta 7.72(\mathrm{~d}, 2 \mathrm{H}, J 8.2 \mathrm{~Hz}, \mathrm{Ar}), 7.53$ (d, 2H, J 8.3 Hz, Ar), 7.30-7.21 (m, 5H, Ar), 7.15-7.12 (m, 5H, Ar), 7.00 (d, J 8.2 Hz, 2H, Ar), 6.96-6.94 (m, 2H, Ar), 5.07 (s, $\left.2 \mathrm{H},-\mathrm{CH}_{2}\right), 4.32\left(\mathrm{~s}, 2 \mathrm{H},-\mathrm{CH}_{2}\right), 2.43\left(\mathrm{~s}, 3 \mathrm{H},-\mathrm{CH}_{3}\right), 2.33\left(\mathrm{~s}, 3 \mathrm{H},-\mathrm{CH}_{3}\right) .{ }^{13} \mathrm{C}-\mathrm{NMR}(125 \mathrm{MHz}$, $\left.\mathrm{CDCl}_{3}\right): \delta 192.3,166.3,144.4,142.1,141.7,140.3,134.5,132.7,129.5,129.3,128.7,128.6$, 128.2, 128.0, 126.4, 126.2, 58.9, 37.0, 21.7, 21.4; IRv $v_{\max }\left(\mathrm{cm}^{-1}\right): 3034,1694,1539,1145,1090$, 726; HRMS-ESI: $[\mathrm{M}+\mathrm{H}]^{+} \mathrm{m} / z$ calcd for $\mathrm{C}_{30} \mathrm{H}_{29} \mathrm{~N}_{2} \mathrm{O}_{3} \mathrm{~S}$ : 497.1899 found: 497.1893 . 
(E)- $\boldsymbol{N}$-(2-Oxopropyl)- $\boldsymbol{N}$,2-diphenyl- $\boldsymbol{N}$ '-tosylacetamidine (4e). Solid (mp 100-102 $\left.{ }^{\circ} \mathrm{C}\right) .{ }^{1} \mathrm{H}$ NMR $\left(500 \mathrm{MHz}, \mathrm{CDCl}_{3}\right) \delta 7.72(\mathrm{~d}, 2 \mathrm{H}, J 7.7 \mathrm{~Hz}, \mathrm{Ar}), 7.27-7.10(\mathrm{~m}, 8 \mathrm{H}, \mathrm{Ar}), 7.00(\mathrm{~d}, J 7.5 \mathrm{~Hz}$, $2 \mathrm{H}, \mathrm{Ar}), 6.87-6.86(\mathrm{~m}, 2 \mathrm{H}, \mathrm{Ar}), 4.43\left(\mathrm{~s}, 2 \mathrm{H},-\mathrm{CH}_{2}\right), 4.25$ (s, 2H, $\left.-\mathrm{CH}_{2}\right), 2.41$ (s, 3H, - $\left.\mathrm{CH}_{3}\right), 2.03$ $\left(\mathrm{s}, 3 \mathrm{H},-\mathrm{CH}_{3}\right) .{ }^{13} \mathrm{C}-\mathrm{NMR}\left(125 \mathrm{MHz}, \mathrm{CDCl}_{3}\right): \delta 201.0,166.4,142.2,141.8,140.4,134.2,129.5$, 129.1, 128.7, 128.5, 128.2, 127.9, 126.4, 62.0, 36.9, 27.3, 21.5; IRv $\max \left(\mathrm{cm}^{-1}\right): 3036,1725,1537$, 1142, 1090, 693; HRMS-ESI: [M+H] ${ }^{+} \mathrm{m} / z$ calcd for $\mathrm{C}_{24} \mathrm{H}_{25} \mathrm{~N}_{2} \mathrm{O}_{3} \mathrm{~S}: 421.1586$ found: 421.1580 .

(E)- $\boldsymbol{N}$-(2-Oxo-2-phenylethyl)- $\boldsymbol{N}$-phenyl-2-pentyl- $\boldsymbol{N}$ '-tosylacetamidine (4f). Oil. ${ }^{1} \mathrm{H}$ NMR (500 $\mathrm{MHz} \mathrm{CDCl}_{3}$ ) $\delta$ 7.76-7.74 (m, 2H, Ar), 7.57-7.53 (m, 3H, Ar), 7.44-7.36 (m, 7H, Ar), 6.98 (d, $2 \mathrm{H}, J 8.1 \mathrm{~Hz}, \mathrm{Ar}), 5.02\left(\mathrm{~s}, 2 \mathrm{H},-\mathrm{CH}_{2}\right), 2.79-2.76\left(\mathrm{~m}, 2 \mathrm{H},-\mathrm{CH}_{2}\right), 2.29\left(\mathrm{~s}, 3 \mathrm{H},-\mathrm{CH}_{3}\right), 1.68-1.62(\mathrm{~m}$, $\left.2 \mathrm{H},-\mathrm{CH}_{2}\right), 1.16-1.02\left(\mathrm{~m}, 6 \mathrm{H},-3 \mathrm{CH}_{2}\right), 0.78\left(\mathrm{t}, 3 \mathrm{H}, J 7.3 \mathrm{~Hz},-\mathrm{CH}_{3}\right) .{ }^{13} \mathrm{C}-\mathrm{NMR}(125 \mathrm{MHz}$, $\left.\mathrm{CDCl}_{3}\right): \delta 192.7,169.2,142.4,141.5,140.7,135.0,133.3,129.8,128.8,128.7,128.5,128.0$, 127.8, 125.9, 58.6, 31.1, 30.7, 29.1, 27.1, 22.2, 21.3, 13.8; IRv $\max \left(\mathrm{cm}^{-1}\right): 2926,1700,1536$, 1145, 1090; HRMS-ESI: $[\mathrm{M}+\mathrm{H}]^{+} m / z$ calcd for $\mathrm{C}_{28} \mathrm{H}_{33} \mathrm{~N}_{2} \mathrm{O}_{3} \mathrm{~S}: 477.2212$ found: 477.2211 .

(E)- $\boldsymbol{N}$-(2-Oxo-2-phenylethyl)- $\boldsymbol{N}$-phenyl-2-p-tolyl- $\boldsymbol{N}$ '-tosylacetamidine (4g). Solid (mp 102$\left.104{ }^{\circ} \mathrm{C}\right) .{ }^{1} \mathrm{H} \mathrm{NMR}\left(500 \mathrm{MHz}, \mathrm{CDCl}_{3}\right) \delta 7.81(\mathrm{~d}, 2 \mathrm{H}, J 7.5 \mathrm{~Hz}, \mathrm{Ar}), 7.58(\mathrm{t}, 1 \mathrm{H}, J 7.2 \mathrm{~Hz}, \mathrm{Ar})$, 7.52 (d, 2H, J 7.9 Hz, Ar), 7.42 (t, 2H, J 7.5 Hz, Ar), 7.29-7.15 (m, 5H, Ar), 6.99 (d, 2H, J 7.8 $\mathrm{Hz}, \mathrm{Ar}), 6.95$ (d, 2H, J 7.7 Hz, Ar), 6.84 (d, 2H, J 7.6 Hz, Ar), 5.09 (s, 2H, -CH $), 4.27$ (s, 2H, $\left.\mathrm{CH}_{2}\right), 2.32\left(\mathrm{~s}, 3 \mathrm{H},-\mathrm{CH}_{3}\right), 2.29\left(\mathrm{~s}, 3 \mathrm{H},-\mathrm{CH}_{3}\right) .{ }^{13} \mathrm{C}-\mathrm{NMR}\left(125 \mathrm{MHz}, \mathrm{CDCl}_{3}\right): \delta 192.8,166.6$, $142.1,141.7,140.4,135.9,135.2$, 133.4, 131.3, 129.5, 128.9, 128.7, 128.6, 128.5, 128.2, 127.9, 126.2, 59.0, 36.5, 21.4, 21.0; IRv $v_{\max }\left(\mathrm{cm}^{-1}\right): 3054,1691,1545,1145,1087,693$; HRMS-ESI: $[\mathrm{M}+\mathrm{H}]^{+} \mathrm{m} / z$ calcd for $\mathrm{C}_{30} \mathrm{H}_{29} \mathrm{~N}_{2} \mathrm{O}_{3} \mathrm{~S}: 497.1899$ found: 497.1881 .

(E)-2-(3-Chlorophenyl)- $N$-(2-oxo-2-phenylethyl)- $N$-phenyl- $N$ '-tosylacetamidine (4h). Solid $\left(\mathrm{mp} \mathrm{108-110}{ }^{\circ} \mathrm{C}\right) .{ }^{1} \mathrm{H} \mathrm{NMR}\left(500 \mathrm{MHz}, \mathrm{CDCl}_{3}\right) \delta$ 7.83-7.81 (m, 2H, Ar), $7.58(\mathrm{t}, J 7.4 \mathrm{~Hz}, 1 \mathrm{H}$, J.7.4 Hz, Ar), 7.52 (d, 2H, J 8.2 Hz, Ar), 7.42 (t, 2H, J 7.8 Hz, Ar), 7.33-7.25 (m, 3H, Ar), 7.157.08 (m, 4H, Ar), 7.01-6.96 (m, 3H, Ar), 6.77 (s, 1H, Ar), 5.10 (s, 2H, $\left.-\mathrm{CH}_{2}\right), 4.30\left(\mathrm{~s}, 2 \mathrm{H},-\mathrm{CH}_{2}\right)$, $2.32\left(\mathrm{~s}, 3 \mathrm{H},-\mathrm{CH}_{3}\right) .{ }^{13} \mathrm{C}-\mathrm{NMR}\left(125 \mathrm{MHz}, \mathrm{CDCl}_{3}\right): \delta 192.5,165.5,142.0,141.8,139.9,136.2$, $135.0,133.9,133.5,129.6,129.5,129.0,128.8,128.7,128.6,128.0,127.9,126.8,126.7,126.1$, 59.0, 36.5, 21.4; HRMS-ESI: IRv $\max \left(\mathrm{cm}^{-1}\right): 3062,1697,1535,1146,1088,687 ;[\mathrm{M}+\mathrm{H}]^{+} \mathrm{m} / z$ calcd for $\mathrm{C}_{29} \mathrm{H}_{26} \mathrm{ClN}_{2} \mathrm{O}_{3} \mathrm{~S}: 517.1353$ found: 517.1339 .

(E)-2-(3-Chlorophenyl)- $N$-(2-oxo-2-p-tolylethyl)- $N$-phenyl- $N$ '-tosylacetamidine (4i). Solid (mp 143-145 ${ }^{\circ} \mathrm{C}$ ). ${ }^{1} \mathrm{H}$ NMR $\left(500 \mathrm{MHz}, \mathrm{CDCl}_{3}\right) \delta 7.73$ (d, 2H, J 8.2 Hz, Ar), 7.52 (d, 2H, J 8.2 $\mathrm{Hz}, \mathrm{Ar}$ ), 7.32-7.26 (m, 3H, Ar), 7.22 (d, 2H, J 8.1 Hz, Ar), 7.14-7.10 (m, 4H, Ar), 7.01 (d, 2H, $J$ 8.1 Hz, Ar), 6.98-6.96 (m, 1H, Ar), 6.76 (s, 1H, Ar), 5.08 (s, 2H, - $\mathrm{CH}_{2}$ ), 4.30 (s, 2H, - $\left.\mathrm{CH}_{2}\right), 2.43$ $\left(\mathrm{s}, 3 \mathrm{H},-\mathrm{CH}_{3}\right), 2.34\left(\mathrm{~s}, 3 \mathrm{H},-\mathrm{CH}_{3}\right) .{ }^{13} \mathrm{C}-\mathrm{NMR}\left(125 \mathrm{MHz}, \mathrm{CDCl}_{3}\right): \delta 192.1,165.5,144.5,141.9$, $140.1,136.3$, 133.9, 132.6, 129.6, 129.5, 129.3, 129.0, 128.8, 128.1, 128.0, 126.8, 126.7, 126.2, 58.9, 36.5, 21.7, 21.4; IR $v_{\max }\left(\mathrm{cm}^{-1}\right):$ 3059, 1692, 1541, 1278, 1145, 1088, 693; HRMS-ESI: $[\mathrm{M}+\mathrm{H}]^{+} \mathrm{m} / \mathrm{z}$ calcd for $\mathrm{C}_{30} \mathrm{H}_{28} \mathrm{ClN}_{2} \mathrm{O}_{3} \mathrm{~S}: 531.1509$ found: 531.1528 .

(E)-2-(3-Chlorophenyl)- $\boldsymbol{N}$-(1-oxo-1-phenylpropan-2-yl)- $N$-phenyl- $N$ '-tosylacetamidine (4j). Solid (mp 140-142 ${ }^{\circ} \mathrm{C}$ ). ${ }^{1} \mathrm{H}$ NMR $\left(500 \mathrm{MHz}, \mathrm{CDCl}_{3}\right) \delta 7.82$ (d, 2H, J 7.4 Hz, Ar), 7.55 (t, 1H, J $7.4 \mathrm{~Hz}, \mathrm{Ar}$ ), 7.45 (d, 2H, J 8.2 Hz, Ar), 7.37-7.19 (m, 6H, Ar), 7.13-7.08 (m, 2H, Ar), 6.92 (d, 
2H, J 8.1 Hz, Ar), 6.88 (d, 2H, J 7.1 Hz, Ar), 6.65 (s, 1H, Ar), 6.05 (q, 1H, J 7.5 Hz, -CH), 4.36 $\left(\mathrm{d}, 1 \mathrm{H}, J 15.4 \mathrm{~Hz},-\mathrm{CH}_{2}\right), 4.09$ (d, $\left.1 \mathrm{H}, J 15.4 \mathrm{~Hz},-\mathrm{CH}_{2}\right), 2.30$ (s, 3H, $\left.-\mathrm{CH}_{3}\right), 0.97$ (d, 3H, J 7.5 $\left.\mathrm{Hz},-\mathrm{CH}_{3}\right) .{ }^{13} \mathrm{C}-\mathrm{NMR}\left(125 \mathrm{MHz}, \mathrm{CDCl}_{3}\right): \delta 198.6,164.9,141.9,139.8,137.2,136.4,135.1$, 133.8, 133.0, 130.9, 130.1, 129.5, 129.2, 129.1, 128.8, 128.7, 128.6, 128.3, 126.7, 126.2, 58.7, 37.7, 21.4, 15.8; IR $v_{\max }\left(\mathrm{cm}^{-1}\right): 3062,1697,1535,1146,1088,688$; HRMS-ESI: $[\mathrm{M}+\mathrm{H}]^{+} \mathrm{m} / \mathrm{z}$ calcd for $\mathrm{C}_{30} \mathrm{H}_{28} \mathrm{ClN}_{2} \mathrm{O}_{3} \mathrm{~S}: 531.1509$ found: 531.1484 .

\section{General procedure for the synthesis of 5}

To a mixture of $4(0.3 \mathrm{mmol})$, silica-gel $(0.5 \mathrm{~g})$ in $\mathrm{CH}_{3} \mathrm{CN}(2 \mathrm{~mL})$ was added DBU $(0.9 \mathrm{mmol})$ under an $\mathrm{N}_{2}$ atmosphere at room temperature. The mixture was stirred at room temperature until 4 consumed (monitored by TLC, usually 1-2h) and then the mixture was elevated to $60{ }^{\circ} \mathrm{C}$ until reaction was completed (monitored by TLC). After evaporation the solvent, the residue was subjected to silica gel column chromatography with petroleum ether/ethyl acetate as eluent to afford pyrroles 5 .

1,3,4-Triphenyl-2-tosylaminopyrrole (5a). Solid (mp 118-120 ${ }^{\circ} \mathrm{C}$ ). ${ }^{1} \mathrm{H}$ NMR (500 MHz, $\left.\mathrm{CDCl}_{3}\right) \delta$ 7.47-7.46 (m, 4H, Ar), 7.39-7.37 (m, 1H, Ar), 7.20-7.06 (m, 10H, Ar), $6.98(\mathrm{~s}, 1 \mathrm{H}$, pyrrole-H), 6.92 (d, J $6.9 \mathrm{~Hz}, 2 \mathrm{H}, \mathrm{Ar}), 6.82(\mathrm{~d}, 2 \mathrm{H}, J 8.1 \mathrm{~Hz}, \mathrm{Ar}), 6.75(\mathrm{br}, 1 \mathrm{H},-\mathrm{NH}), 2.29$ (s, $\left.3 \mathrm{H},-\mathrm{CH}_{3}\right) .{ }^{13} \mathrm{C}-\mathrm{NMR}\left(125 \mathrm{MHz}, \mathrm{CDCl}_{3}\right): \delta 142.9,138.8,136.3,134.8,133.4,129.8,129.1$, $128.1,128.0,127.9,127.2,126.9,126.0,125.8,125.7,123.5,122.2,120.3,119.9,21.4 ; \mathrm{IR} v_{\max }$ $\left(\mathrm{cm}^{-1}\right): 3263,1598,1499,1390,1160,754,697$; HRMS-ESI: $[\mathrm{M}+\mathrm{H}]^{+} \mathrm{m} / \mathrm{z}$ calcd for $\mathrm{C}_{29} \mathrm{H}_{25} \mathrm{~N}_{2} \mathrm{O}_{2} \mathrm{~S}: 465.1637$ found: 465.1633 .

1-(4-Methylphenyl)-3,4-diphenyl-2-tosylaminopyrrole (5b). Solid (mp 158-160 $\left.{ }^{\circ} \mathrm{C}\right) .{ }^{1} \mathrm{H}$ NMR $\left(500 \mathrm{MHz}, \mathrm{CDCl}_{3}\right) \delta 7.35(\mathrm{~d}, J .8 .2 \mathrm{~Hz}, 2 \mathrm{H}, \mathrm{Ar}), \delta 7.24(\mathrm{~d}, J 8.2 \mathrm{~Hz}, 2 \mathrm{H}, \mathrm{Ar}), 7.20-7.06(\mathrm{~m}, 10 \mathrm{H}$, Ar), 6.95 (s, 1H, pyrrole-H), 6.93-6.92 (m, 2H, Ar), 6.82 (d, 2H, J $8.0 \mathrm{~Hz}, \mathrm{Ar}), 6.78$ (br, 1H, $\mathrm{NH}), 2.43\left(\mathrm{~s}, 3 \mathrm{H},-\mathrm{CH}_{3}\right), 2.29\left(\mathrm{~s}, 3 \mathrm{H},-\mathrm{CH}_{3}\right) .{ }^{13} \mathrm{C}-\mathrm{NMR}\left(125 \mathrm{MHz}, \mathrm{CDCl}_{3}\right): \delta 142.8,137.0$, 136.5, 136.3, 134.9, 133.4, 129.8, 129.7, 129.0, 128.1, 128.0, 127.9, 126.9, 125.9, 125.8, 125.6, 123.2, 122.0, 120.4, 119.9, 21.4, 21.1; IRv $v_{\max }\left(\mathrm{cm}^{-1}\right): 3251,1598,1499,1390,1160,754,697$; HRMS-ESI: $[\mathrm{M}+\mathrm{H}]^{+} \mathrm{m} / \mathrm{z}$ calcd for $\mathrm{C}_{30} \mathrm{H}_{27} \mathrm{~N}_{2} \mathrm{O}_{2} \mathrm{~S}$ : 479.1793 found: 479.1788 .

1-(2-Methylphenyl)-3,4-diphenyl-2-tosylaminopyrrole (5c). Solid (mp 148-150 $\left.{ }^{\circ} \mathrm{C}\right) .{ }^{1} \mathrm{H}$-NMR $\left(500 \mathrm{MHz}, \mathrm{CDCl}_{3}\right): \delta$ 7.37-7.28 (m, 4H, Ar), 7.20-7.07 (m, 10H, Ar), 6.95 (d, 2H, J 6.9 Hz, Ar), 6.88 (br, 1H, -NH), 6.85-6.83 (m, 3H, Ar \& pyrrole-H), 2.31 (s, 3H, - $\left.\mathrm{CH}_{3}\right), 2.25\left(\mathrm{~s}, 3 \mathrm{H},-\mathrm{CH}_{3}\right)$. ${ }^{13} \mathrm{C}-\mathrm{NMR}\left(125 \mathrm{MHz}, \mathrm{CDCl}_{3}\right): \delta 142.8,137.6,136.5,135.6,135.0,133.6,130.7,130.0,129.1$, 128.8, 128.3, 128.1, 128.0, 127.9, 126.9, 126.3, 125.9, 125.7, 123.0, 121.0, 120.1, 21.4, 17.9; $\mathrm{IR} v_{\max }\left(\mathrm{cm}^{-1}\right): 3266,1605,1386,1159,754,697$; HRMS-ESI: $[\mathrm{M}+\mathrm{H}]^{+} \mathrm{m} / \mathrm{z}$ calcd for $\mathrm{C}_{30} \mathrm{H}_{27} \mathrm{~N}_{2} \mathrm{O}_{2} \mathrm{~S}$ : 479.1793 found: 479.1788 .

1,3-Diphenyl-4-(4-methylphenyl)-2-tosylaminopyrrole (5d). Solid (mp 178-180 $\left.{ }^{\circ} \mathrm{C}\right) .{ }^{1} \mathrm{H}-\mathrm{NMR}$ $\left(500 \mathrm{MHz}, \mathrm{CDCl}_{3}\right): \delta$ 7.47-7.45 (m, 4H, Ar), 7.38-7.35 (m, 1H, Ar); $7.16(\mathrm{~d}, 2 \mathrm{H}, J 8.3 \mathrm{~Hz}, \mathrm{Ar})$, 7.11-7.06 (m, 3H, Ar), 7.01 (s, 4H, Ar), 6.95 (s, 1H, pyrrole-H), 6.91-6.90 (m, 2H, Ar), $6.82(\mathrm{~d}$, $2 \mathrm{H}, J 8.1 \mathrm{~Hz}, \mathrm{Ar}), 6.66(\mathrm{br}, 1 \mathrm{H},-\mathrm{NH}), 2.30\left(\mathrm{~s}, 3 \mathrm{H},-\mathrm{CH}_{3}\right), 2.28\left(\mathrm{~s}, 3 \mathrm{H},-\mathrm{CH}_{3}\right) .{ }^{13} \mathrm{C}-\mathrm{NMR}(125$ $\left.\mathrm{MHz}, \mathrm{CDCl}_{3}\right): \delta 142.9,138.8,136.2,135.4,133.4,131.8,129.8,129.1,128.9,127.9,127.1$, 
126.9, 126.0, 125.7, 123.4, 122.1, 120.2, 119.7, 21.4, 21.0; IR $v_{\max }\left(\mathrm{cm}^{-1}\right): 3264,1598,1499$, 1388, 1329, 1161, 754, 697; HRMS-ESI: $[\mathrm{M}+\mathrm{H}]^{+} \mathrm{m} / \mathrm{z}$ calcd for $\mathrm{C}_{30} \mathrm{H}_{27} \mathrm{~N}_{2} \mathrm{O}_{2} \mathrm{~S}: 479.1793$ found: 479.1779.

1,3-Diphenyl-4-methyl-2-tosylaminopyrrole (5e). Solid (mp 108-110 $\left.{ }^{\circ} \mathrm{C}\right) .{ }^{1} \mathrm{H}-\mathrm{NMR}(500 \mathrm{MHz}$, $\mathrm{CDCl}_{3}$ ): $\delta$ 7.44-7.31 (m, 5H, Ar), 7.18-7.16 (m, 5H, Ar); 7.05-7.03 (m, 2H, Ar), $6.82(\mathrm{~d}, 2 \mathrm{H}, J$ $8.2 \mathrm{~Hz}, \mathrm{Ar}), 6.75(\mathrm{br}, 1 \mathrm{H},-\mathrm{NH}), 6.67\left(\mathrm{q}, 1 \mathrm{H}, J 0.7 \mathrm{~Hz}\right.$, pyrrole-H), $2.31\left(\mathrm{~s}, 3 \mathrm{H},-\mathrm{CH}_{3}\right), 2.05$ (s, $\left.3 \mathrm{H},-\mathrm{CH}_{3}\right) .{ }^{13} \mathrm{C}-\mathrm{NMR}\left(125 \mathrm{MHz}, \mathrm{CDCl}_{3}\right): \delta 142.8,138.9,136.4,133.9,129.2,129.0,127.9$, 127.0, 126.7, 125.8, 125.7, 123.7, 119.9, 118.8, 117.1, 21.4, 11.2; IRv $v_{\max }\left(\mathrm{cm}^{-1}\right): 3250,1598$, 1501, 1390, 1160, 754, 697; HRMS-ESI: $[\mathrm{M}+\mathrm{H}]^{+} \mathrm{m} / \mathrm{z}$ calcd for $\mathrm{C}_{24} \mathrm{H}_{23} \mathrm{~N}_{2} \mathrm{O}_{2} \mathrm{~S}: 403.1480$ found: 403.1467.

1,4-Diphenyl-3-pentyl-2-tosylaminopyrrole (5f). Solid (mp 100-102 $\left.{ }^{\circ} \mathrm{C}\right) .{ }^{1} \mathrm{H}-\mathrm{NMR}(500 \mathrm{MHz}$, $\left.\mathrm{CDCl}_{3}\right): \delta$ 7.43-7.36 (m, 6H, Ar), 7.28-7.25 (m, 4H, Ar); 7.08-7.04 (m, 4H, Ar), $6.77(\mathrm{~s}, 1 \mathrm{H}$, pyrrole-H), 6.54 (br, $1 \mathrm{H},-\mathrm{NH}), 2.51-2.48\left(\mathrm{~m}, 2 \mathrm{H},-\mathrm{CH}_{2}\right), 2.39\left(\mathrm{~s}, 3 \mathrm{H},-\mathrm{CH}_{3}\right), 1.38-1.35(\mathrm{~m}, 2 \mathrm{H},-$ $\left.\mathrm{CH}_{2}\right), 1.23-1.17\left(\mathrm{~m}, 4 \mathrm{H},-\mathrm{CH}_{2}\right), 0.83\left(\mathrm{t}, 3 \mathrm{H}, J 7.1 \mathrm{~Hz},-\mathrm{CH}_{3}\right) .{ }^{13} \mathrm{C}-\mathrm{NMR}\left(125 \mathrm{MHz}, \mathrm{CDCl}_{3}\right): \delta$ 143.3, 138.5, 136.9, 136.0, 129.3, 128.9, 128.4, 127.7, 127.2, 126.6, 126.0, 125.2, 123.9, 122.9, 119.5, 119.3, 32.0, 29.6, 24.4, 22.3, 21.5, 14.0; $\operatorname{IRv} v_{\max }\left(\mathrm{cm}^{-1}\right): 2952,1597,1499,1390,1160$, 754, 697; HRMS-ESI: [M+H] ${ }^{+} \mathrm{m} / \mathrm{z}$ calcd for $\mathrm{C}_{28} \mathrm{H}_{31} \mathrm{~N}_{2} \mathrm{O}_{2} \mathrm{~S}: 459.2106$ found: 459.2094 .

1,4-Diphenyl-3-(4-methylphenyl)-2-tosylaminopyrrole (5g). Solid (mp 204-206 $\left.{ }^{\circ} \mathrm{C}\right) .{ }^{1} \mathrm{H}-\mathrm{NMR}$ $\left(500 \mathrm{MHz}, \mathrm{CDCl}_{3}\right): \delta$ 7.49-7.44 (m, 4H, Ar), 7.39-7.36 (m, 1H, Ar); 7.21-7.14 (m, 7H, Ar), 6.97(s, 1H, pyrrole-H), 6.88 (d, 2H, J 7.9 Hz, Ar), $6.83(\mathrm{~d}, 2 \mathrm{H}, J 8.2 \mathrm{~Hz}, \mathrm{Ar}), 6.79$ (d, 2H, J 8.1 $\mathrm{Hz}, \mathrm{Ar}), 6.66(\mathrm{br}, 1 \mathrm{H},-\mathrm{NH}), 2.32\left(\mathrm{~s}, 3 \mathrm{H},-\mathrm{CH}_{3}\right), 2.31\left(\mathrm{~s}, 3 \mathrm{H},-\mathrm{CH}_{3}\right) .{ }^{13} \mathrm{C}-\mathrm{NMR}(125 \mathrm{MHz}$, $\left.\mathrm{CDCl}_{3}\right): \delta 142.9,138.8,136.5,135.6,134.9,130.3,129.6,129.1,128.9,128.6,128.1,128.0$, 127.1, 127.0, 125.8, 125.7, 123.4, 122.1, 120.3, 119.8, 21.5, 21.2; IRv $v_{\max }\left(\mathrm{cm}^{-1}\right): 3249,1597$ 1501, 1400, 1328, 1161, 754, 697; HRMS-ESI: $[\mathrm{M}+\mathrm{H}]^{+} \mathrm{m} / \mathrm{z}$ calcd for $\mathrm{C}_{30} \mathrm{H}_{27} \mathrm{~N}_{2} \mathrm{O}_{2} \mathrm{~S}: 479.1793$ found: 479.1780 .

1,4-Diphenyl-3-(3-chlorophenyl)-2-tosylaminopyrrole (5h). Solid (mp 216-218 $\left.{ }^{\circ} \mathrm{C}\right) .{ }^{1} \mathrm{H}-\mathrm{NMR}$ (500 MHz, $\left.\mathrm{CDCl}_{3}\right): \delta$ 7.49-7.47 (m, 4H, Ar), 7.42-7.39 (m, 1H, Ar); 7.24-7.16 (m, 5H, Ar), 7.12$7.10(\mathrm{~m}, 2 \mathrm{H}, \mathrm{Ar}), 7.07-7.05(\mathrm{~m}, 1 \mathrm{H}, \mathrm{Ar}), 7.00(\mathrm{t}, 1 \mathrm{H}, J 7.9 \mathrm{~Hz}, \mathrm{Ar}), 6.98(\mathrm{~s}, 1 \mathrm{H}$, pyrrole-H), 6.89 $(\mathrm{d}, 2 \mathrm{H}, J 8.1 \mathrm{~Hz}, \mathrm{Ar}), 6.85-6.83(\mathrm{~m}, 2 \mathrm{H}, \mathrm{Ar}), 6.72(\mathrm{br}, 1 \mathrm{H},-\mathrm{NH}), 2.32\left(\mathrm{~s}, 3 \mathrm{H},-\mathrm{CH}_{3}\right) .{ }^{13} \mathrm{C}-\mathrm{NMR}$ $\left(125 \mathrm{MHz}, \mathrm{CDCl}_{3}\right): \delta 143.3,138.5,136.4,135.3,134.3,133.8,129.6,129.2,129.1,128.2,128.1$, 128.0, 127.5, 126.9, 126.1, 125.9, 123.4, 120.7, 120.5, 120.2, 21.5; IRv $v_{\max }\left(\mathrm{cm}^{-1}\right): 3255,1597$, 1500, 1390, 1160, 754, 697; HRMS-ESI: $[\mathrm{M}+\mathrm{H}]^{+} \mathrm{m} / \mathrm{z}$ calcd for $\mathrm{C}_{29} \mathrm{H}_{24} \mathrm{ClN}_{2} \mathrm{O}_{2} \mathrm{~S}: 499.1247$ found: 499.1233 .

1-Phenyl-3-(3-chlorophenyl)-4-(4-methylphenyl)-2-tosylaminopyrrole (5i). Solid (mp 163$\left.165{ }^{\circ} \mathrm{C}\right) .{ }^{1} \mathrm{H}-\mathrm{NMR}\left(500 \mathrm{MHz}, \mathrm{CDCl}_{3}\right): \delta$ 7.50-7.45 (m, 4H, Ar), 7.40-7.37 (m, 1H, Ar); $7.22(\mathrm{~d}, J$ $8.2 \mathrm{~Hz}, 2 \mathrm{H}, \mathrm{Ar}), 7.11$ (br, 1H, -NH), 7.06-6.97 (m, 6H, Ar), 6.94 (s, 1H, pyrrole-H), 6.88-6.85 (m, 4H, Ar), $2.31\left(\mathrm{~s}, 6 \mathrm{H},-2 \mathrm{CH}_{3}\right) .{ }^{13} \mathrm{C}-\mathrm{NMR}\left(125 \mathrm{MHz}, \mathrm{CDCl}_{3}\right): \delta$ 143.2, 138.6 136.5, 135.7, 135.5, 133.7, 131.4, 129.6, 129.1, 129.0, 128.2, 127.9, 127.3, 126.8, 126.0, 125.8, 123.3, 120.8, 120.4, 119.9, 21.5, 21.1; IR $v_{\max }\left(\mathrm{cm}^{-1}\right): 3248,1597,1500,1391,1160,754,697$; HRMS-ESI: $[\mathrm{M}+\mathrm{H}]^{+} \mathrm{m} / \mathrm{z}$ calcd for $\mathrm{C}_{30} \mathrm{H}_{26} \mathrm{ClN}_{2} \mathrm{O}_{2} \mathrm{~S}: 513.1404$ found: 513.1392 . 
1,4-Diphenyl-3-(3-chlorophenyl)-4-methyl-2-tosylaminopyrrole (5j). Solid (mp 210-212 $\left.{ }^{\circ} \mathrm{C}\right)$. ${ }^{1} \mathrm{H}-\mathrm{NMR}\left(500 \mathrm{MHz}, \mathrm{CDCl}_{3}\right.$ ): $\delta$ 7.53-7.46 (m, 3H, Ar), 7.40-7.38 (m, 2H, Ar), 7.28-7.18 (m, 5H, Ar), 7.06-7.05 (m, 2H, Ar), 6.94-6.93 (m, 1H, Ar), 6.88-6.84 (m, 3H, Ar), 6.80 (t, J 1.6 Hz, 1H, $\mathrm{Ar}), 6.72(\mathrm{~d}, 1 \mathrm{H}, J 7.7 \mathrm{~Hz}, \mathrm{Ar}), 2.28\left(\mathrm{~s}, 3 \mathrm{H},-\mathrm{CH}_{3}\right), 2.08\left(\mathrm{~s}, 3 \mathrm{H},-\mathrm{CH}_{3}\right) .{ }^{13} \mathrm{C} \mathrm{NMR}(125 \mathrm{MHz}$, CDC13): $\delta 143.0,136.9,136.8,135.8,134.9,133.4,130.4,129.5,129.0,128.9,128.8,128.2$, $128.0,127.9,127.1,126.8,125.8,125.3,120.4,120.2,119.3,21.4,12.0 ; \operatorname{IR} v_{\max }\left(\mathrm{cm}^{-1}\right): 3225$, 1598, 1499, 1381, 1162, 761, 694; HRMS-ESI: $[\mathrm{M}+\mathrm{H}]^{+} \mathrm{m} / \mathrm{z}$ calcd for $\mathrm{C}_{30} \mathrm{H}_{26} \mathrm{ClN}_{2} \mathrm{O}_{2} \mathrm{~S}: 513.1404$ found: 513.1399 .

\section{Acknowledgements}

Financial support from the Natural Science Foundation of Zhejiang Province (Y4100662) and the Opening Foundation of Zhejing Provincial Top Key Discipline is greatly appreciated.

\section{References and Notes}

1. For reviews on pyrroles, see: (a) Gribble, G. W. In Comprehensive Heterocyclic Chemistry; Katritzky, A. R., Rees, C. W., Scriven, E. F. V., Eds.; Pergamon: Oxford. 1996; Vol. 2, p 207. (b) Jones, R. A. Pyrroles, Part II, The Synthesis, Reactivity and Physical Properties of Substituted Pyrroles; Wiley: New York, 1992. (c) Gilchrist, T. L. Heterocyclic Chemistry, 3rd Ed.; Addison-Wesley Longman: Essex. 1997; p 192. (d) Joule, J. A.; Mills, K. In Heterocyclic Chemistry; Blackwell Science: Oxford, 2000; Chapter 13. (e) Fürstner, A. Angew. Chem., Int. Ed. 2003, 42, 3582. (f) Hoffmann, H.; Lindel, T. Synthesis 2003, 1753. (g) Balme, G. Angew. Chem., Int. Ed. 2004, 43, 6238.

2. For reviews of the pyrrole structure in materials, see: (a) Electronic Materials: The Oligomer Approach; Müllen, K., Wegner, G., Eds.; Wiley-VCH: Weinheim, 1997. (b) Matano, Y.; Imahori, H. Acc. Chem. Res. 2009, 42, 1193. (c) Higgins, S. Chem. Soc. Rev. 1997, 26, 247.

3. (a) Knorr, L. Ber. 1884, 17, 1635. For recent examples: (b) Shiner, C. M.; Lash, T. D. Tetrahedron 2005, 61, 11628. (c) Bellingham, R. K.; Carey, J. S.; Hussain, N.; Morgan, D. O.; Oxley, P.; Powling, L. C. Org. Process Res. Dev. 2004, 8, 279. (d) Manley, J. M.; Kalman, M. J.; Conway, B. G.; Ball, C. C.; Havens, J. L.; Vaidyanathan, R. J. Org. Chem. 2003, 68, 6447 .

4. (a) Hantzsch, A. Ber. 1890, 23, 1474. For recent examples: (b) Matiychuk, V. S.; Martyak, R. L.; Obushak, N. D.; Ostapiuk, Y. V.; Pidlypnyi, N. I. Chem. Heterocycl. Comp. 2004, 40, 1218. (c) Calvo, L.; González-Ortega, A.; Sañudo, M. C. Synthesis 2002, 2450. (d) Trautwein, A. W.; Süßmuth, R. D.; Jung, G. Bioorg. Med. Chem. Lett. 1998, 8, 2381. 
5. (a) Paal, C. Ber. 1885, 18, 367. (b) Knorr, L. Ber. 1885, 18, 299. For recent examples: (c) Chen, J.; Wu, H.; Zheng, Z.; Jin, C.; Zhang, X.; Su, W. Tetrahedron Lett. 2006, 47, 5383. (d) Minetto, G.; Raveglia, L. F.; Sega, A.; Taddei, M. Eur. J. Org. Chem. 2005, 5277. (e) Banik, B. K.; Banik, I.; Renteria, M.; Dasgupta, S. K. Tetrahedron Lett. 2005, 46, 2643.

6. For reviews on pyrrole synthesis, see: (a) Sundberg, R. J. In Comprehensive Heterocyclic Chemistry; Katritzky, A. R., Rees, C. W., Scriven, E. F. V., Eds.; Pergamon: Oxford, 1996; Vol. 2, p 119. (b) Gilchrist, T. L. J. Chem. Soc., Perkin Trans. 1 1999, 2849. (c) Balme, G. Angew. Chem., Int. Ed. 2004, 43, 6238. (d) Trofimov, B. A. In Adv. Heterocycl. Chem.; Katritzky, A. R., Ed.; Preparation of Pyrroles from Ketoximes and Acetylenes. Academic: San Diego, 1990; Vol. 51, pp 177-301. (e) Trofimov, B. A. In The Chemistry of Heterocyclic Compounds; Part 2; Pyrroles; Jones, R. A., Ed.; Vinylpyrroles; Wiley: New York, 1992; Vol 48, pp 131-298. (f) Trofimov, B. A.; Mikhaleva, A. I.; Schmidt, E. Y.; Sobenina, L. N. In Adv. Heterocycl. Chem.; Pyrroles and N-vinylpyrroles from Ketones and Acetylenes: Recent Strides 2010, Vol. 99, Chapter 7, pp 209-254. (g) Ferreira, V. F.; de Souza, M. C. B. V.; Cunha, A. C.; Pereira, L. O. R.; Ferreira, M. L. G. Org. Prep. Proced. Int. 2001, 33, 411. (h) Estèvez, V.; Villacampa, M.; Menéndez, J. C. Chem. Soc. Rev. 2010, 39, 4402.

7. For selected recent references, see: (a) Narayan, R.; Fröhlich, R.; Würthwein, E. U. J. Org. Chem. 2012, 77, 1868. (b) Chiba, S.; Wang, Y. F.; Lapointe, G.; Narasaka, K. Org. Lett. 2008, 10, 313. (c) Peng, L.; Zhang, X.; Ma, J.; Zhong, Z.; Wang, J. Org. Lett. 2007, 9, 1445. (d) Rivero, M. R.; Buchwald, S. L. Org. Lett. 2007, 9, 973. (e) Dong, C.; Deng, G.; Wang, J. J. Org. Chem. 2006, 71, 5560. (f) Fuchibe, K.; Ono, D.; Akiyama, T. Chem. Commun. 2006, 2271. (g) St. Cyr, D. J.; Martin, N.; Arndtsen, B. A. Org. Lett. 2007, 9, 449. (h) Huang, X.; Shen, R.; Zhang, T. J. Org. Chem. 2007, 72, 1534. (i) Bélanger, G.; April, M.; Dauphin, É.; Roy, S. J. Org. Chem. 2007, 72, 1104. (j) Lu, L.; Chen, G.; Ma, S. Org. Lett. 2006, 8, 835. (k) Ackermann, L.; Sandmann, R.; Kaspar, L. T. Org. Lett. 2009, 11, 2031. (1) Cież, D. Org. Lett. 2009, 11, 4282. (m) Davies, P. W.; Martin, N. Org. Lett. 2009, 11, 2293. (n) Cyr, D. J. S.; Martin, N.; Arndtsen, B. A. Org. Lett. 2007, 9, 449. (o) Jr, W. R. D.; Zheng, Z. J. Org. Chem. 2009, 74, 5626. (p) Misra, N. C.; Panda, K.; Ila, H.; Junjappa, H. J. Org. Chem. 2007, 72, 1246. (q) Bergner, I.; Opatz, T. J. Org. Chem. 2007, 72, 7083. (r) Verniest, G.; Claessens, S.; Kimpe, N. D. Tetrahedron 2005, 61, 4631. (s) Tehrani, K. A.; Borremans, D.; Kimpe, N. D. Tetrahedron 1999, 55, 4133. (t) Aelterman, W.; Kimpe, N. D.; Tyvorskii, V.; Kulinkovich, O. J. Org. Chem. 2001, 66, 53.

8. (a) Rostovtsev, V. V.; Green, L. G.; Fokin, V. V.; Sharpless, K. B. Angew. Chem., Int. Ed. 2002, 41, 2596. (b) Tornøe, C. W.; Christensen, C.; Meldal, M. J. Org. Chem. 2002, 67, 3057. (c) Yoo, E. J.; Ahlquist, M.; Bae, I.; Sharpless, K. B.; Fokin, V. V.; Chang, S. J. Org. Chem. 2008, 73, 5520.

9. (a) Bae, I.; Han, H.; Chang, S. J. Am. Chem. Soc. 2005, 127, 2038. (b) Cho, S. H.; Yoo, E. J.; Bae, I.; Chang, S. J. Am. Chem. Soc. 2005, 127, 16046. (c) Cho, S. H.; Chang, S. Angew. Chem., Int. Ed. 2007, 46, 1897. (d) Yoo, E. J.; Chang, S. Org. Lett. 2008, 10, 1163. (e) Cho, S. H.; Chang, S. Angew. Chem., Int. Ed. 2008, 47, 2836. (f) Kim, J.; Lee, Y.; Lee, J.; Do, Y.; 
Chang, S. J. Org. Chem. 2008, 73, 9454. (g) Yoo, E. J.; Park, S. H.; Lee, S. H.; Chang, S. Org. Lett. 2009, 11, 1155. (h) Cui, S. L.; Lin, X. F.; Wang, Y. G. Org. Lett. 2006, 8, 4517. (i) Cui, S. L.;Wang, J.; Wang, Y. G. Org. Lett. 2007, 9, 5023. (j) Lu, W.; Song,W. Z.; Hong, D.; Lu, P.;Wang, Y. G. Adv. Synth. Catal. 2009, 351, 1768. (k) Jin, H.W.; Xu, X. L.; Gao, J. R.; Zhong, J. H.; Wang, Y. G. Adv. Synth. Catal. 2010, 352, 347. (1) Cassidy, M. P.; Raushel, J.; Fokin, V. V. Angew. Chem., Int. Ed. 2006, 45, 3154. (m) Whiting, M.; Fokin, V. V. Angew. Chem., Int. Ed. 2006, 45, 3157; (n) Xu, X.; Cheng, D.; Li, J.; Guo, H.; Yan, J. Org. Lett. 2007, 9, 1585.

10. (a) Chen, W.; Cui, J.; Zhu, Y.; Hu, X.; Mo, W. J. Org. Chem. 2012, 77, 1585. (b) Chen, W.; Li, J.; Zhu, Y.; Ye, L.; Hu, W.; Mo, W. Arkivoc 2011, (ix), 381.(c) Chen, W.; Su, C.; Huang, X. Synlett 2006, 1446. (d) Chen, W.; Huang, X.; Zhou, H.; Ren, L. Synthesis 2006, 609. (e) Chen, W.; Li, J.; Zhu, Y.; Ye, L.; Hu, W.; Mo, W. Arkivoc 2012, (vi), 16.

11. Synthesis of 2-aminopyrrole derivatives: Verhé, R.; Kimpe, N. D.; Buyck, L. D.; Tilley, M.; Schamp, N. Tetrahedron 1980, 36, 131.

12. (a) Chinchilla, R.; Najera, C.; Yus, M. Chem. Rev. 2004, 104, 2667. (b) Tanabe, Y.; Wakimura, K.; Nishii, Y.; Muroya, Y. Synthesis 1996, 388. (c) Hooper, M. W.; Utsunomiya, M.; Hartwig, J. F. J. Org. Chem. 2003, 68, 2861. (d) Padwa, A.; Crawford, K. R.; Rashatasakhon, P.; Rose, M. J. Org. Chem. 2003, 68, 2609. (e) Crawford, K. R.; Padwa, A. Tetrahedron Lett. 2002, 43, 7365.

13. The structure was assigned on the basis of its ${ }^{1} \mathrm{H}$ NMR, ${ }^{13} \mathrm{C}$ NMR, IR spectra, MS data and through the comparative configuration analysis of $4 \mathbf{i}$. The configuration of $4 \mathbf{i}$ is $E$-isomer, which has been established on the basis of 2D NOESY experiment, see supporting information.

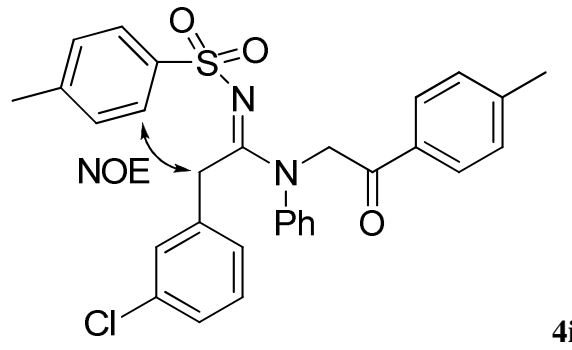

14. One kind of reaction product with high polarity could be monitored firstly during the reaction using thin layer chromatography. We think this may be the alcohol intermediates. We could separate this alcohol intermediate with partially converted to pyrrole using preparative TLC. It showed that silica-gel could promote the reaction. ${ }^{1} \mathrm{H}$ NMR $(500 \mathrm{MHz}$, DMSO-d $\left.d_{6}\right) \delta .74(\mathrm{~d}, 2 \mathrm{H}, J 7.9 \mathrm{~Hz}, \mathrm{Ar}), 7.52$ (t, 2H, J 8.0 Hz, Ar), 7.35 (t, 1H, J 7.4 Hz, Ar), 7.26-7.25 (m, 2H, Ar), 7.16-7.03 (m, 10H, Ar), 6.76 (d, 2H, J 7.3 Hz, Ar), 6.31 (br, 1H, $\mathrm{OH}), 5.09(\mathrm{~s}, 1 \mathrm{H},-\mathrm{CH}), 5.05\left(\mathrm{~d}, 1 \mathrm{H}, J 11.6 \mathrm{~Hz},-\mathrm{CH}_{2}\right), 3.91\left(\mathrm{~d}, 1 \mathrm{H}, J 11.6 \mathrm{~Hz},-\mathrm{CH}_{2}\right), 2.28$ $\left(\mathrm{s}, 3 \mathrm{H},-\mathrm{CH}_{3}\right) .{ }^{13} \mathrm{C}-\mathrm{NMR}\left(125 \mathrm{MHz}, \mathrm{DMSO}-d_{6}\right): \delta 167.7,141.4,140.0,139.5,138.6,134.3$, $128.8,128.7,128.4,127.9,127.3,127.1,126.7,126.5,126.3,125.4,123.6,79.0,62.1,59.9$, 20.7 . 
15. (a) Lakner, F. J.; Parker, M. A.; Rogovoy, B.; Khvat, A.; Ivachtchenko, A. Synthesis 2009, 1987. (b) Hattori, G.; Sakata, K.; Matsuzawa, H.; Tanabe, Y.; Miyake Y.; Nishibayashi, Y. J. Am. Chem. Soc. 2010, 132, 10592. 